\title{
A High Precision Stray-immune Weak Capacitance Measurement System for Gas- solid Two-phase Flows Detection
}

\author{
Feihu Song, Hui Hong, Shuai Liu \\ Institute of Microelectronic CAD, Hangzhou Dianzi University, Hangzhou, 310018, China
}

\begin{abstract}
A novel weak capacitance measurement system is proposed to measure the gas-solid two-phase flows. The system is mainly consist of capacitance sensor, Microprogrammed Control Unit (MCU), Direct Digital Synthesizer (DDS), operational amplifier, analogue multiplier and low-pass filter. Since the stray capacitance is much larger than the variation of measured capacitance, the stray capacitance should be eliminate by the measurement system. The experimental results show that the system meets the application requirement, and has good linearity, high measurement accuracy and stray-immune. The result of the percentage error is about $0.6 \%$ and the non-linear error is less than $1.5 \%$. The sampling rate which is related to the frequency of the signal generator and the MCU clock can reach 500 kps.
\end{abstract}

Keywords-capacitance measurement; weak capacitance; direct digital synthesizer; stray-immune.

\section{INTRODUCTION}

With the development of science and technology, gassolid two-phase flows systems are widely used in agricultural production and modern industrial. The measuring solid phase's concentration is a technical difficulty in pneumatic transmission systems. The gassolid two-phase flows systems are uncontrollable and complex. On the other hand, the flows can not be uniform-flow [1]. Many systems have been introduced to convert the solid's concentration to a DC voltage. However, most systems are not able to hand small variations of the concentration. The charge/discharge capacitance measurement circuit system is simple and low cost, but has drift problem and extremely slow response speed [2]. The electrostatic method based on electrostatic induction principle is vulnerable to the influence of the solid physical characteristics [3]. One method is based on the frequency modulation circuit which has high sensitivity, but the system is susceptible to external interference [4].

Among methods referred above, the capacitance method is more suitable for the gas-solid two-phase flows system. However, the capacitance variation of capacitive sensor is extremely small. The stray capacitance is much larger than the nominal sensor's capacitance variation. Therefore, how to improve the measurement sensitivity and SNR (signal to noise ratio) becomes an important problem in weak capacitance measurement system.

This paper presents a novel capacitance measurement system which consisted of a new capacitive sensor and a detection circuit. The capacitive sensor has the advantages of simple structure, high precision, and reliable work etc. The circuit is stray-immune and can reject undesired common mode interference. What's more, its sample speed is extremely high.

\section{CAPACITANCE Measurement System}

\section{A. Principle description}

The flow character of the two-phase flows is shown in Fig.1 which gives the placement of the capacitance sensor's two measurement electrodes. A sine-wave is applied to one electrode of the capacitive sensor, and the other electrode would generate an induction signal. Then the induction signal is amplified and demodulated to obtain a DC signal representing the unknown capacitance. When the solid flow in the capacitance sensor, the DC signal would follow suit, and the solid phase's concentration of the gas-solid two-phase flows system is obtained.

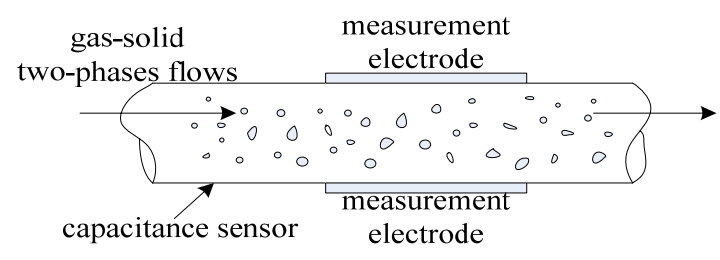

Figure 1. The flow character of the two-phase flows

\section{B. Schematic Diagram}

The capacitance measurement system is shown in Fig.2. 


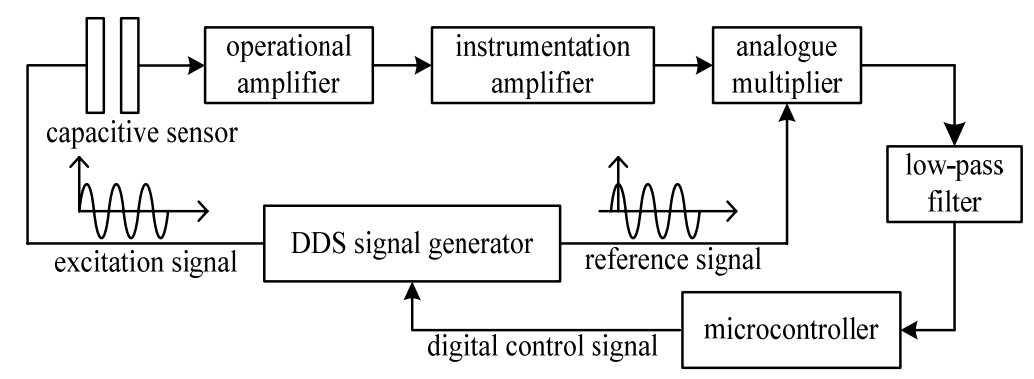

Figure 2. The capacitance measurement system

With the digital control signal, the DDS is used to generate two sine-wave signals. One signal is used as an excitation signal and the other as a reference signal. When one of the sensor's electrodes received the excitation signal, the other electrode would generate an induction signal which is small AC current.

Because the output of capacitance sensor is a quite small current signal, an operational amplifier and an instrumentation amplifier are used to convert the $\mathrm{AC}$ current into an AC voltage. In order to suppress the noise and improve the SNR, an analogue multiplier is used to demodulate the voltage and the reference signal. The reference signal is the other sine-wave signal of the DDS.

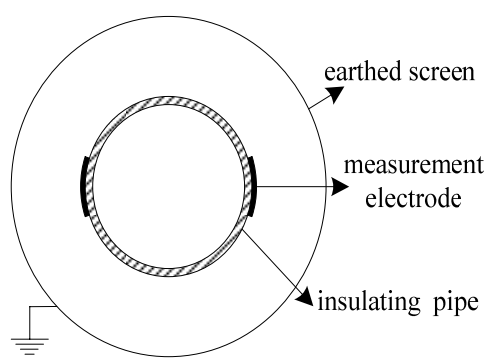

(a) Typical capacitive sensor

Figure 3. The two-dimensional profiles of typical and new capacitive sensor

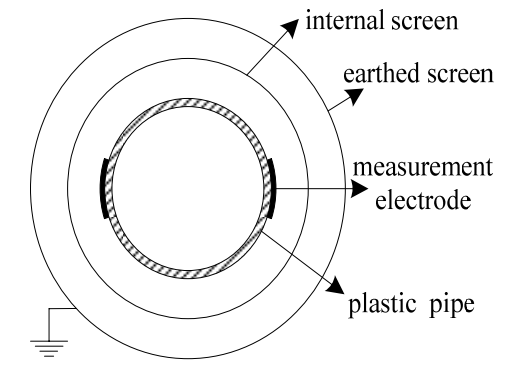

(b) New capacitive sensor
A low-pass filter is used to cut off the AC signal and take up the useful DC signal.

\section{Capacitance Sensor}

The two-dimensional profiles of two different structure capacitive sensors are shown in Fig.3. Two measurement electrodes mounted symmetrically of a plastic pipe. An earthed screen, which surrounds the plastic pipe, can reduce the external electrical interference signal. The new capacitive sensor is proposed to reduce the external noises as much as possible (see Fig.3). An internal screen between the plastic pipe and the earthed screen, which is made of copper and plastic, is used to minimize external electrical noises. The new sensor can achieve much better effect than the typical sensor.

\section{CIRCUIT DESIGN}

\section{A. Analog Circuit Unit}

The Analog Circuit Unit consists of signal generator, amplifier, analogue multiplier and low-pass filter. A sinewave is applied to the capacitive sensor, producing an AC output signal. Then the AC signal is amplified and demodulated to obtain a DC signal representing the unknown capacitance.

The DDS chip AD9854 as the signal generator is selected ${ }^{[5]}$.The DDS's output signal expression is shown as follows:

$$
u_{e}(t)=u_{r}(t)=U \sin (2 \pi f t)
$$

where $U$ is the amplitude of the signal, $f$ is the frequency of the sine-wave signal.

An operational amplifier AD549 and an instrumentation amplifier AD620 are used to convert AC current into an AC voltage. The output of AD549 is given by

$$
\begin{aligned}
u_{i} & =-G j \omega C_{x} R u_{e} \\
& =-G j \omega C_{x} R U \sin (2 \pi f t)
\end{aligned}
$$

Where $\mathrm{G}$ is the gain of $\mathrm{AD620}, \omega$ is the angular frequency of the excitation signal, $\mathrm{C}_{\mathrm{x}}$ is the measurement capacitance.

The AC voltage is demodulated by the analogue multiplier AD734. The signal is multiplied in a translinear core of novel design to generate the product. 
The demodulated signal consists of a sine-wave and a DC voltage. A low-pass filter LTC 1164-6 is used to reject the sine-wave signal. The final resultant DC voltage represents the unknown capacitance. Fig.4 is the analog circuit unit.

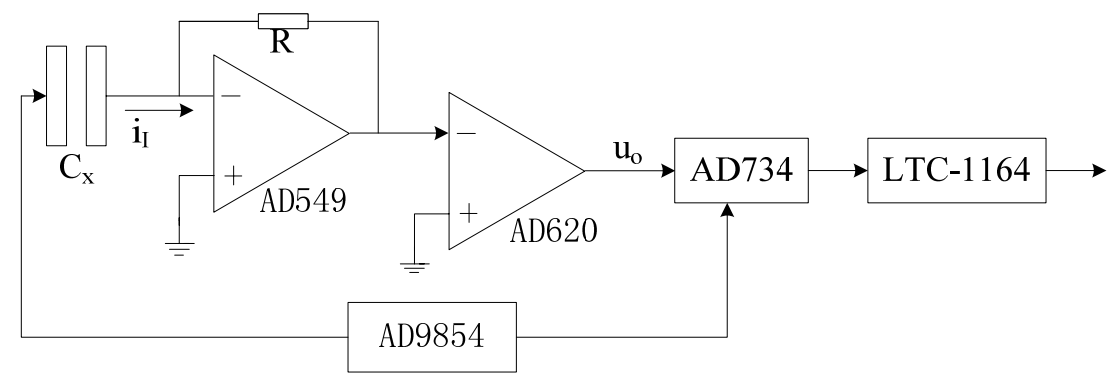

Figure 4. The Analog Circuit Unit

\section{B. Circuit Control Unit}

The circuit control unit consists of $\mathrm{MCU}$ and its peripheral circuit. The MSP430F149 is used as the MCU. The circuit unit is shown in Fig.5. The software flow-chat is shown in Fig.6. After the digital control signal is input to the DDS, the system starts to work. The MCU reads results using the interrupt method in the procession of data processing.

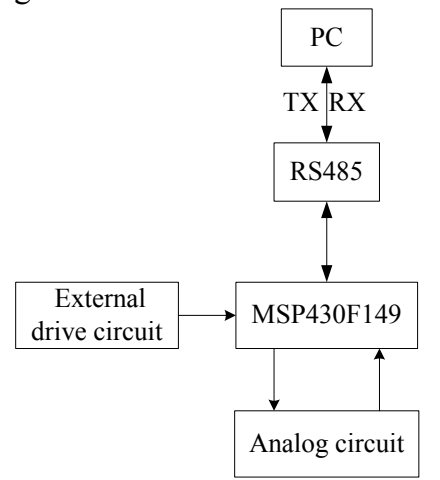

Figure 5. The MCU and its peripheral circuit

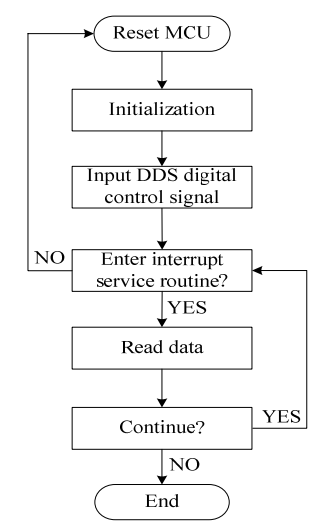

Figure 6 . The software flow-chat

\section{EXPERIMENTAL RESULTS}

In this section, the experiment is delivered to show the precision and performance of the weak capacitance measurement system. Fig. 7 is the physical diagram of the experimental system. It is composed of the capacitance sensor and the capacitance measurement circuit.

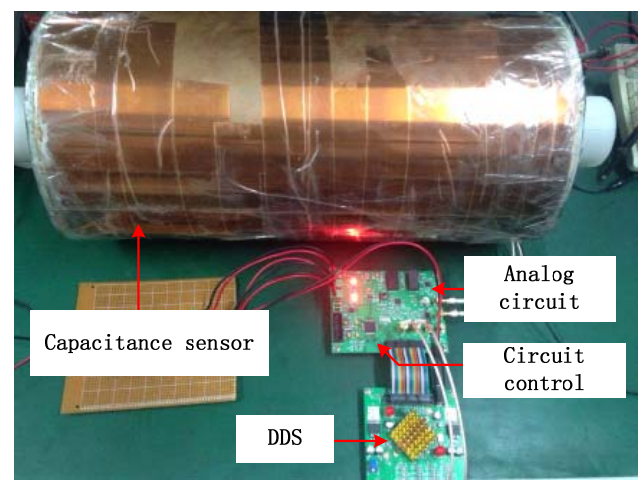

Figure 7. The physical diagram of the system

Fig. 8 illustrates 1000 measurements of the capacitance sensor. The experiment results show that the minimum value was $1.342 \mathrm{pF}$, the maximum value was $1.357 \mathrm{pF}$ and the average value was $1.3495 \mathrm{pF}$. The maximum absolute error was only $0.0075 \mathrm{pF}$. The result of the percentage error is about $0.6 \%$. The experiments testing capacitance measurement system indicate that it meets the demands of industrial well. 


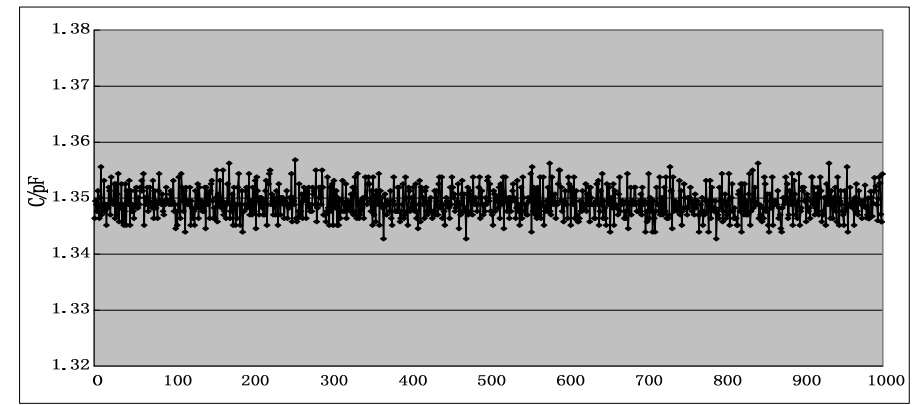

Figure 8. Experimental values of the new capacitance sensor

The dynamic response of the system is delivered by using different volume of the pulverized coal. The corresponding values of the capacitor are obtained with the proportionate increase of solid's concentration. The experimental results are shown in Fig.9.

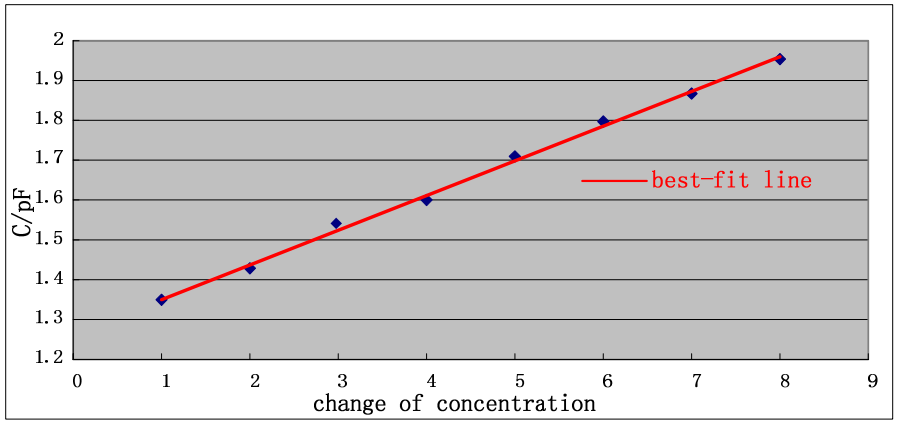

Figure 9. The dynamic experimental values of the new capacitance sensor

It shows clearly in the Fig.9 that the output capacitance is basically a linear with the concentration change. The non-linear error is about $1.474 \%$. The test results show that the system agrees with the requirements of project at the stability, reliability, real-time.

\section{CONCLUSIONS}

In this paper, a new weak capacitance measurement system is presented, which can be used to measure the gas-solid two-phase flows. It is composed of a new capacitance sensor and a detection circuit. The measured method is used in analog method, and its data acquisition speed is faster than the methods. Its non-linear error is quite small.

The system can reduce the parasitic and stray capacitance, and it also suppresses the electromagnetic interference by adopting the shielded electric cables and double-shielded capacitance. The experimental results show that the capacitance measurement system has merits of rapid sampling, high precision of operation results and high reliability.

\section{ACKNOWLEDGMENT}

In this paper, the research was sponsored by the Nature Science Foundation of China (Project No. 61107025) and the Key Innovation Team Project of Zhejiang Province (Project No.2010R50010).

\section{REFERENCES}

[1] Wang Yan, Lu De-cai, Li Rui, Research for Measuring Concentration of Gas/Solid Two phases Flow. The Tenth International Conference on Electronic Measurement \& Instruments, pp. 346-349,2011.

[2] Kjaersgaard-Rasmussen, J\& Yang, W.Q. A compact electrical capacitance tomography system. IEEE International Workshop on Imaging Systems and Techniques, pp.175-180,2008.

[3] Tianming Chen, Bowler N\& Bowler,J.R. Analysis of Arc-Electrode Capacitive Sensors for Characterization of Dielectric Cylindrical Rods. IEEE Instrumentation and Measurement, 61(1), pp.233-240, 2012.

[4] Ji-Tzuoh Lin\& Kevin W.W, Douglas Jackson. Development of capacitive pure bending strain sensor for wireless spinal fusion monitoring. Sensors and Actuators A, 138(2), pp. 276-287, 2007.

[5] Shi Yu Yan\& Ji Zhou Li. Research on the DDS' CPLD Control to Generate Special Band Signal. IEEE BioMedical Engineering and Informatics, 2, pp. 681-684, 2008. 